Original paper

\title{
Three-dimensional printer-generated patient-specific phantom for artificial in vivo dosimetry in radiotherapy quality assurance
}

\author{
Takeshi Kamomae ${ }^{\mathrm{a}, *}$, Hidetoshi Shimizu ${ }^{\mathrm{b}}$, Takayoshi Nakaya ${ }^{\mathrm{c}}$, Kuniyasu Okudaira ${ }^{\mathrm{c}}$, \\ Takahiro Aoyama ${ }^{\mathrm{b}}$, Hiroshi Oguchi ${ }^{\mathrm{d}}$, Masataka Komori ${ }^{\mathrm{d}}$, Mariko Kawamura ${ }^{\mathrm{e}}$, \\ Kazuhiro Ohtakara ${ }^{\mathrm{e}}$, Hajime Monzen ${ }^{\mathrm{f}}$, Yoshiyuki Itoh ${ }^{\mathrm{a}}$, Shinji Naganawa ${ }^{\mathrm{e}}$ \\ a Department of Therapeutic Radiology, Nagoya University Graduate School of Medicine, 65 Tsurumai-cho, Showa-ku, Nagoya 466-8550, Japan \\ b Department of Radiation Oncology, Aichi Cancer Center Hospital, 1-1 Kanokoden, Chikusa-Ku, Nagoya 464-8681, Aichi, Japan \\ c Department of Radiological Technology, Nagoya University Hospital, 65 Tsurumai-cho, Showa-ku, Nagoya 466-8560, Japan \\ d Department of Radiological and Medical Laboratory Sciences, Nagoya University Graduate School of Medicine, 1-1-20 Daiko-Minami, Higashi-ku, Nagoya 461-8673, \\ Japan \\ e Department of Radiology, Nagoya University Graduate School of Medicine, 65 Tsurumai-cho, Showa-ku, Nagoya 466-8550, Japan \\ ${ }^{\mathrm{f}}$ Department of Medical Physics, Graduate School of Medical Science, Kindai University, 377-2 Ohno-Higashi, Osaka-Sayama, Osaka 589-8511, Japan
}

\section{A R T I C L E I N F O}

\section{Keywords:}

3D printer

In vivo dosimetry

IMRT QA

\begin{abstract}
A B S T R A C T
Pretreatment intensity-modulated radiotherapy quality assurance is performed using simple rectangular or cylindrical phantoms; thus, the dosimetric errors caused by complex patient-specific anatomy are absent in the evaluation objects. In this study, we construct a system for generating patient-specific three-dimensional (3D)printed phantoms for radiotherapy dosimetry. An anthropomorphic head phantom containing the bone and hollow of the paranasal sinus is scanned by computed tomography (CT). Based on surface rendering data, a patient-specific phantom is formed using a fused-deposition-modeling-based 3D printer, with a polylactic acid filament as the printing material. Radiophotoluminescence glass dosimeters can be inserted in the 3D-printed phantom. The phantom shape, CT value, and absorbed doses are compared between the actual and 3D-printed phantoms. The shape difference between the actual and printed phantoms is less than $1 \mathrm{~mm}$ except in the bottom surface region. The average CT value of the infill region in the 3D-printed phantom is $-6 \pm 18$ Hounsfield units (HU) and that of the vertical shell region is $126 \pm 18 \mathrm{HU}$. When the same plans were irradiated, the dose differences were generally less than $2 \%$. These results demonstrate the feasibility of the 3D-printed phantom for artificial in vivo dosimetry in radiotherapy quality assurance.
\end{abstract}

\section{Introduction}

Intensity-modulated radiation therapy (IMRT) and volumetricmodulated arc therapy (VMAT) have recently been adopted for various cancer sites, contributing to improvements in survival outcomes with fewer treatment-related side effects [1-4]. Previous reports recommend that patient-specific quality assurance (QA) be performed for patients having IMRT and VMAT to reduce the likelihood of dose errors and assure patient safety during radiotherapy $[5,6]$. Detecting delivered dose errors in IMRT and VMAT is especially important, as these involve complex procedures that contain many potential errors. In general, patient-specific QA is performed on the direct dose measurements or independent dose calculation. Independent dose calculation systems have recently progressed rapidly; one of their limitations, however, is its lack of validation of the deliverability of a treatment plan on the actual treatment device [7]. Therefore, these validations must be performed in combination with thorough quality control of accelerator and multileaf collimator performance. Moreover, it is strongly recommended to perform experimental verification for a relatively large number of patients to prevent systematic dose errors during initial clinical implementation of a new IMRT technique [8]. For this reason, based on institutional policies or protocols, patient-specific QA is often conducted using experimental dose measurements. Experimental dose measurements are usually performed using simple rectangular or cylindrical phantoms with various detectors (e.g., an ionization chamber or radiochromic film) [9-11]. In some cases, pretreatment QA results may reveal disagreement between the measured and treatment planning system (TPS)-calculated phantom doses. In these cases, we need to judge whether these disagreements are at acceptance or action levels by considering other metric values, such as the distance to the agreement

\footnotetext{
* Corresponding author.

E-mail address: kamomae@med.nagoya-u.ac.jp (T. Kamomae).
} 
1. CT image acquisition
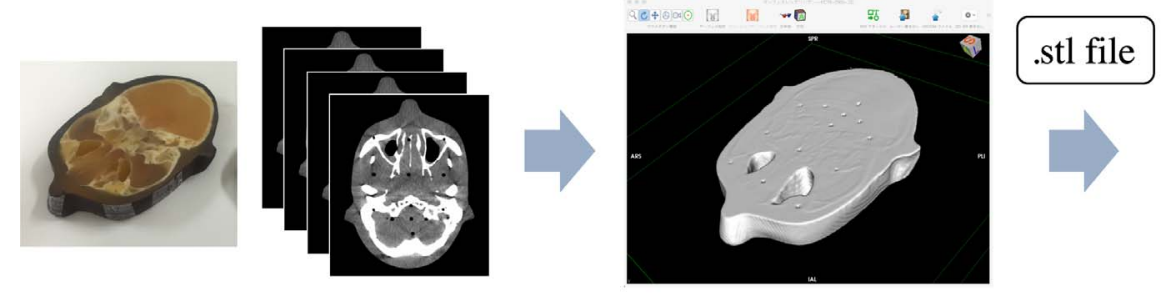

Printing parameters

\section{Printing}

(Ninjabot FDM-200W)
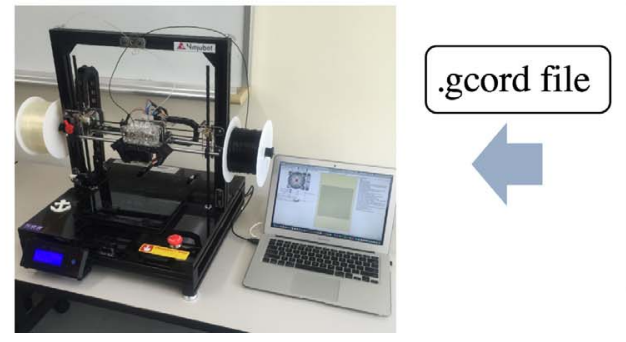

\section{Preparation for} insertion of dosimeters

(Shade3D)
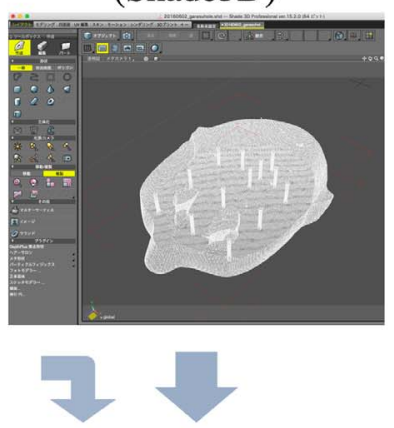

4. Slicing

(Slic3r)

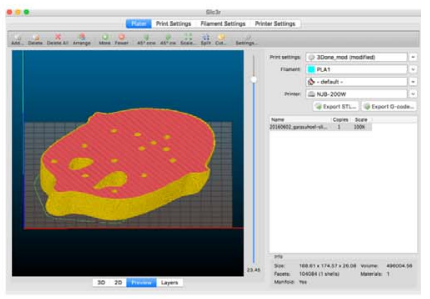

Fig. 1. Fabrication overview of a patient-specific 3D-printed phantom for dosimetry.

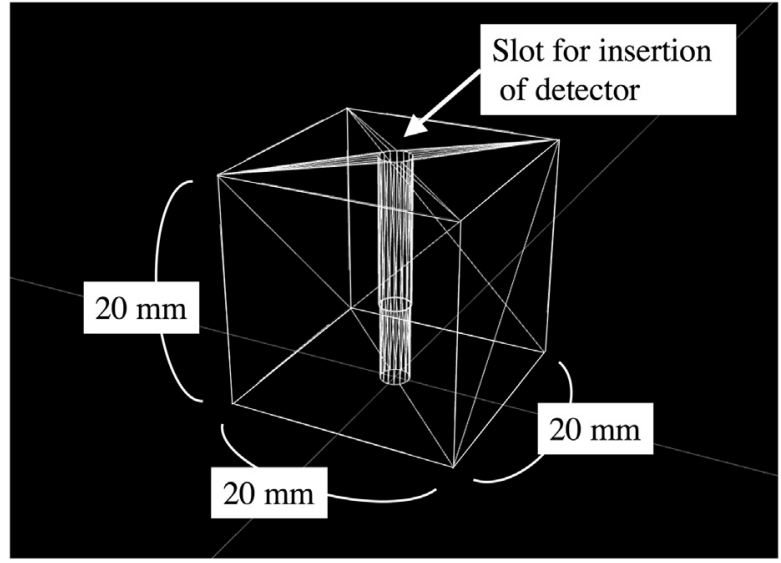

Fig. 2. Trial 3D model for evaluating the slot diameter for RPL glass dosimeter insertion; slot diameters of $2.8 \mathrm{~mm}, 2.9 \mathrm{~mm}, 3.0 \mathrm{~mm}$, and $3.1 \mathrm{~mm}$ were validated.

Table 1

Printing parameters.

\begin{tabular}{ll}
\hline Settings & Values \\
\hline Bed temperature & $60^{\circ} \mathrm{C}$ \\
Extruder temperature & $195^{\circ} \mathrm{C}$ \\
Layer height & $0.3 \mathrm{~mm}$ \\
First layer height & $0.35 \mathrm{~mm}$ \\
Vertical shells & 4 \\
Horizontal shells & 4 \\
Speed of print movement (Perimeter) & $60 \mathrm{~mm} / \mathrm{s}$ \\
Speed of print movement (External perimeter) & $30 \mathrm{~mm} / \mathrm{s}$ \\
Speed of print movement (Infill) & $80 \mathrm{~mm} / \mathrm{s}$ \\
First layer print movement speed & $30 \mathrm{~mm} / \mathrm{s}$ \\
Fill density & $100 \%$ \\
Fill pattern & Rectilinear \\
Fill angle & $45^{\circ}$ \\
Nozzle diameter & $0.4 \mathrm{~mm}$ \\
Filament diameter & $1.75 \mathrm{~mm}$ \\
\hline
\end{tabular}

and gamma index. However, recent work has discussed the insufficient sensitivity of these conventional pretreatment QA results in predicting the clinical impact on the patient from changes in the dose-volume histograms for the clinical target volume and organs at risk $[12,13]$. One reason for this poor sensitivity is that the shape of the phantoms used for pretreatment QA and that of patients are entirely different. In fact, it is difficult to estimate the magnitude and location of a dose error in a patient from the phantom dose error. Ideally, the magnitude and location of the errors in the patient may provide more important information than the evaluation metrics (e.g., pass rate) based on conventional pretreatment QA, and would be more critical in clinical judgment. From these viewpoints, to estimate the in vivo dose to the patient is a challenge in pretreatment QA.

Recent studies have demonstrated that 3D printing offers flexibility in design and manufacturing for auxiliary equipment in radiotherapy [14]. Ju et al. suggested a new system for manufacturing a range compensator in proton therapy, using an industrial 3D printer with ultraviolet curable acrylic plastic [15]. Park et al. fabricated a patientspecific customized bolus, using a low-cost personal 3D printer with a polylactic acid (PLA) filament, and evaluated its feasibility for posttotal-mastectomy electron conformal therapy [16]. Further, Ehler et al. demonstrated the construction of a patient-specific phantom, using a 3D printer with an acrylonitrile butadiene styrene (ABS) filament for dosimetry [17]. ABS warps on cooling, preventing this phantom from being printed as a $100 \%$ solid object; thus, their approach was to print the exterior shape of the phantom and fill it with a uniform mixture of a modified M3 mix.

PLA filaments are generally more robust to warping during cooling than ABS filaments $[16,18,19]$. The aim of this study is to construct a system for the fabrication of patient-specific phantoms, using a 3D printer with a PLA filament and to evaluate the feasibility of this 3Dprinted phantom for artificial in vivo dosimetry in radiotherapy QA. To simplify this procedure for clinical use, the phantom is fabricated using only 3D printing - without filling the inner cavity with a liquid material such as the modified M3 mix. Considering the fabrication cost of the phantom, we have adopted a low-cost personal 3D printer. 

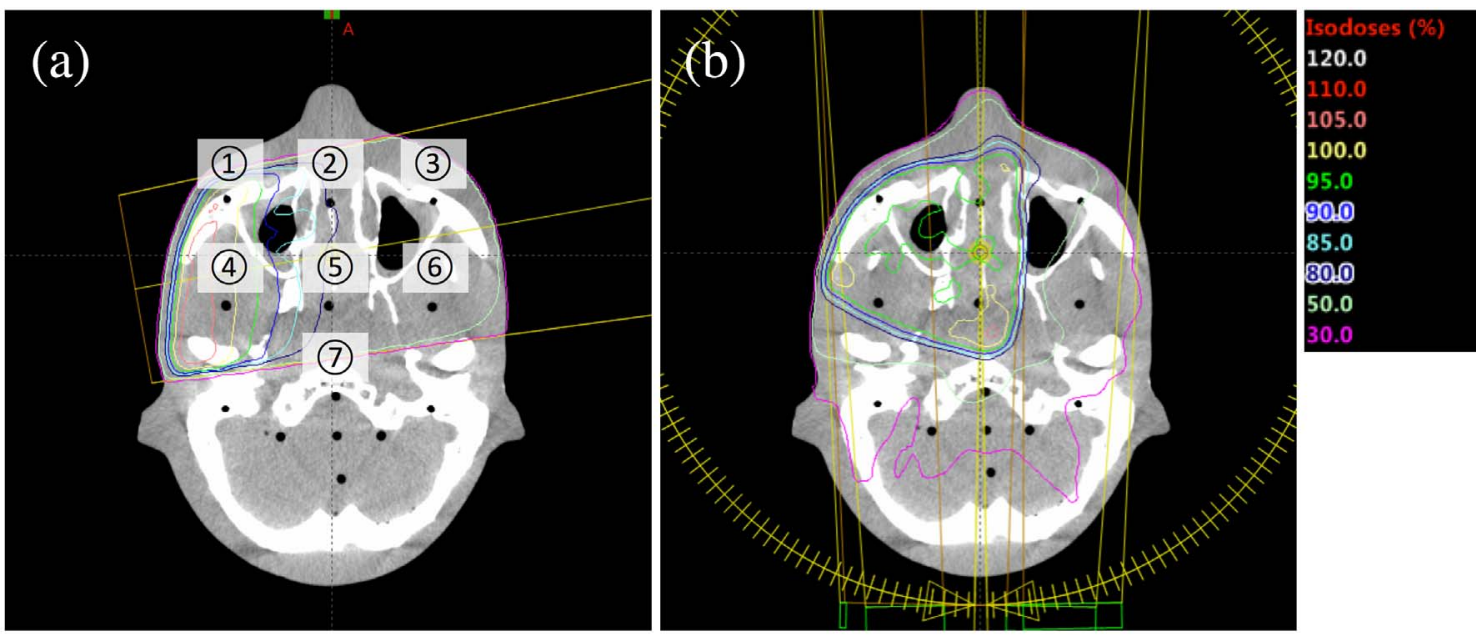

Fig. 3. Positional relationships between the irradiation beam and the RPL glass dosimeter slots (circled numbers, 1-7) for (a) a single static beam and (b) a two-arc VMAT plan.

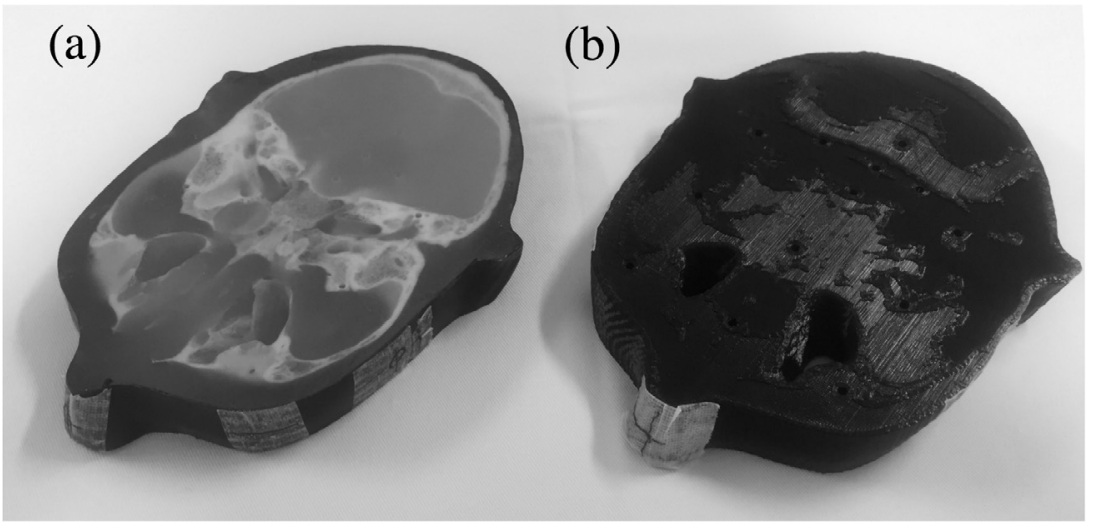

Fig. 4. Image of the (a) anthropomorphic head phantom containing the bone and hollow of the paranasal sinus and (b) the 3D-printed phantom.

\section{Materials and methods}

\subsection{Image acquisition, data processing, and printing procedure}

The overall fabrication process is summarized in Fig. 1. An anthropomorphic head phantom containing the bone and hollow of the maxillary sinus (QualitA Co, Ltd., Nagano, Japan) was selected as the mock patient. The fabrication target object was one section of the phantom (Fig. 1, process 1), and the maximum lengths were about $25 \mathrm{~mm}, 150 \mathrm{~mm}$, and $195 \mathrm{~mm}$ in the superior-inferior, left-right, and anterior-posterior directions, respectively. The phantom was scanned using a computed tomography (CT) scanner (Asteion 4; Toshiba Medical Systems, Tokyo, Japan). The CT data were reconstructed in a 300$\mathrm{mm}$ field of view on a $512 \times 512$ grid with a slice thickness of $1.0 \mathrm{~mm}$ and exported in DICOM format.

Data processing was performed per the following three steps: (i) creation of the 3D surface rendering of the exterior shape of the patient, (ii) formation of the insertion slots for dosimeters, and (iii) creation of the control code containing the build information for 3D printing.

OsiriX Lite ver. 8.0.2 (Pixmeo Inc., Bernex, Switzerland) was used for 3D surface rendering of the patient's exterior shape. The threshold value for surface rendering was set to -500 Hounsfield units (HU), which matches the OsiriX default surface rendering value of the skin surface and that of previous research [17]. Surface rendering data were saved in a stereo-lithography (STL) format file.

The STL file was imported into 3D modeling software (Shade3D professional ver. 15; Shade3D Co., Ltd., Tokyo, Japan) to prepare slots for dosimeter insertion. A GD-302 M radiophotoluminescence (RPL) glass dosimeter (Asahi Techno Glass Corporation, Shizuoka, Japan) and an FGD-1000 readout system (Asahi Techno Glass Corporation, Shizuoka, Japan) were selected for the dosimeter for several reasons: the uniformity and reproducibility of the signal from 50 RPL glass dosimeters are each $\pm 1.1 \%$ (one standard deviation); good dose linearity is maintained for doses ranging up to $30 \mathrm{~Gy}$; energy dependence is within $2 \%$ for photon energies of a ${ }^{60} \mathrm{Co}$ beam, $4 \mathrm{MV}$, and $10 \mathrm{MV}$ X-ray beams; and angular dependence, ranging from $0^{\circ}$ (along the long axis of the RPL glass dosimeter) to $120^{\circ}$, is within $1.5 \%$ [20]. RPL glass dosimeters also exhibit less fading (within $1.7 \%$ after five months) and can be read repeatedly [21]. The diameter of the RPL glass dosimeter, stored in a dedicated plastic holder, was measured with a micrometer (NSK Ltd, Tokyo, Japan) as $2.78 \mathrm{~mm}$. Prior to final 3D printing, trial printing to determine the slot diameter was performed because contraction was expected to occur during cooling. Trial 3D models were also created with Shade3D software, an example of which is depicted in Fig. 2. The exterior shape was a $20 \mathrm{~mm} \times 20 \mathrm{~mm} \times 20 \mathrm{~mm}$ cube; slot diameters of $2.8 \mathrm{~mm}, 2.9 \mathrm{~mm}, 3.0 \mathrm{~mm}$, and $3.1 \mathrm{~mm}$ were validated.

The STL files were then compiled into G-code, which is used to run the commands that modulate the position, velocity, temperature, and extrusion timing of the 3D printer, using Slic3r Ver. 1.2.9 [22] slicing software; the printing parameters are summarized in Table 1.

The patient-specific phantom was generated based on the G-code data using a FDM-200W personal 3D printer (Ninjabot, LCC, Shizuoka, Japan) with a PLA filament, which is a fused-deposition-modeling (FDM)-based 3D printer.

\subsection{Irradiation plans and evaluation methods}

In this study, all measurement are based on the use of 6-MV photon 

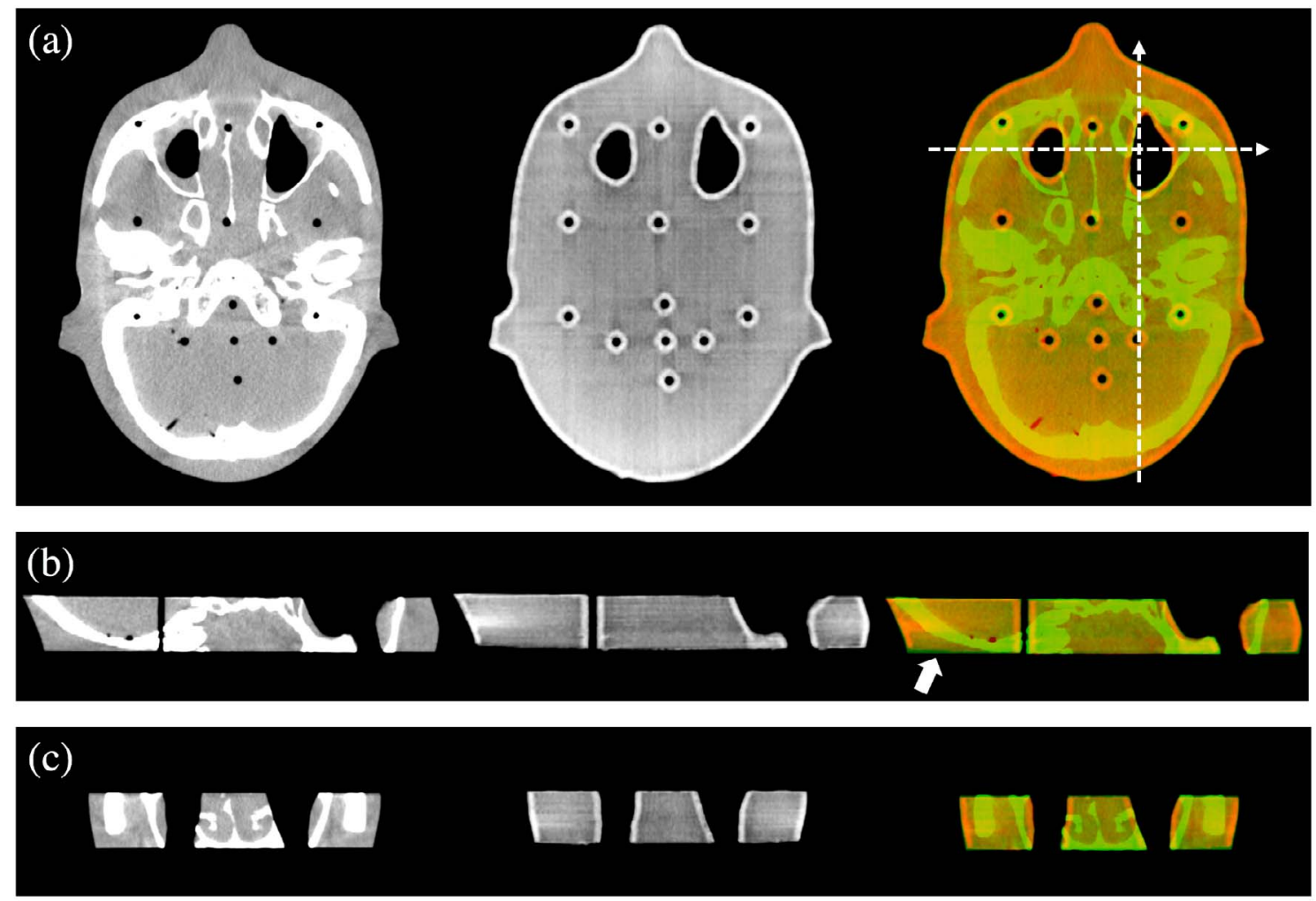

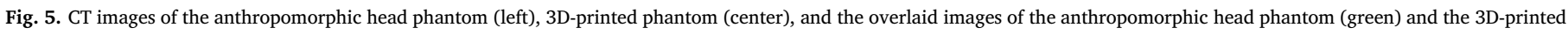
phantom (red) (right): (a) axial, (b) coronal, and (c) sagittal views. The white arrow represents region in which the geometric error exceeds 1 mm.

beams produced using a Clinac iX linear accelerator (Varian Medical Systems, Palo Alto, CA). To evaluate basic properties, the phantoms were irradiated using a single beam with a $260^{\circ}$ gantry angle and a field size of $8 \mathrm{~cm} \times 8 \mathrm{~cm}$. The monitor unit was set to $100 \mathrm{MU}$. The positional relationships between the irradiation beam and the RPL glass dosimeters are shown in Fig. 3a. The dose distributions were calculated with Eclipse TPS (Ver. 13.6; Varian Medical System, Palo Alto, CA). All dose calculations were performed using the anisotropic analytical algorithm (AAA; Varian Medical Systems, Palo Alto, CA) and a dose calculation grid size of $1.0 \mathrm{~mm} \times 1.0 \mathrm{~mm}$. In this study, to minimize the angular dependence of the RPL glass dosimeter, all dosimeters were placed perpendicular to the beam axis, and their readout centers coincided with the isocenter plane of the linear accelerator.

A clinical case was evaluated using a two-arc VMAT plan. A maxillary sinus cancer patient (coinciding with the anatomical site of the 3D-printed phantom) who had previously undergone VMAT at our institution was selected. The study was approved by the Institutional Review Board of the Nagoya University Hospital and all patients provided written informed consent. Two-arc VMAT was performed using $181^{\circ}-179^{\circ}$ clockwise and $179^{\circ}-181^{\circ}$ counterclockwise rotations; the positional relationships between the irradiation beam and the RPL glass dosimeters are depicted in Fig. 3b.

Dose readings of the RPL glass dosimeters were performed automatically. We determined the calibration rate between the average raw output value of ten RPL glass dosimeters and the dose measured by the calibrated ionization chamber (Farmer-type ionization chamber type 30013; PTW, Freiburg, Germany). This calibration was performed using the 6-MV photon beam with a $10 \mathrm{~cm} \times 10 \mathrm{~cm}$ field size in a solid water phantom (Tough Water, Kyoto Kagaku Co., Ltd., Kyoto, Japan), at a depth of $10 \mathrm{~cm}[20,23]$. The final outputs were obtained by multiplying the raw output values of the RPL glass dosimeters by the calibration rate. To eliminate the fading effect, the readout was performed within $24 \mathrm{~h}$ after irradiation. Measurements of the single static beam and the two-arc VMAT plan were repeated thrice.
To evaluate the feasibility of using the 3D-printed phantom for dosimetry, the phantom shape and CT value were compared with the anthropomorphic head phantom. Furthermore, the similarity of their external shapes was determined by calculating their Dice similarity coefficient [24]. These evaluations were performed using Eclipse TPS. The average CT value was calculated from 20 randomly selected points of interest. The dose differences, obtained from the RPL glass dosimeters, between the anthropomorphic head and 3D-printed phantoms were then estimated. Finally, the differences between the measured and TPS-calculated doses were determined. Moreover, in one case, the CT values of the 3D-printed phantom were converted to $374 \mathrm{HU}$, which corresponds to the PLA density of $1.25 \mathrm{~g} / \mathrm{cm}^{3}$, and dose comparisons were continued.

\section{Results}

In a trial print to verify the slot diameter for inserting the RPL glass dosimeters, slot diameters of $2.8 \mathrm{~mm}, 2.9 \mathrm{~mm}, 3.0 \mathrm{~mm}$, and $3.1 \mathrm{~mm}$ were tested; the best size in our environment was $3.0 \mathrm{~mm}$. Diameters of $2.8 \mathrm{~mm}$ and $2.9 \mathrm{~mm}$ could not be inserted into and $3.1 \mathrm{~mm}$ was loose.

Fig. 4 shows images of the anthropomorphic head and 3D-printed phantoms. The total printing time for this part was approximately $20 \mathrm{~h}$. The shape difference between the anthropomorphic head phantom and the 3D-printed phantom was less than $1 \mathrm{~mm}$, except for the bottom surface region (Fig. 5). The white arrows in Fig. 5b represent geometric errors exceeding $1 \mathrm{~mm}$. A maximum difference of $2.0 \mathrm{~mm}$ was exhibited on the bottom surface of the posterior region in the patient. The Dice similarity coefficient for the external shape was 0.974 . A comparison of the CT value profiles is depicted in Fig. 6. Except for the bone region, the profile shape of the 3D-printed phantom coincided with that of the anthropomorphic head phantom. The maximum difference in CT values for the internal region was within $\pm 100 \mathrm{HU}$, and that of the border region between tissue and air was within $\pm 250 \mathrm{HU}$. The average CT value of the soft tissue region in the anthropomorphic head phantom 

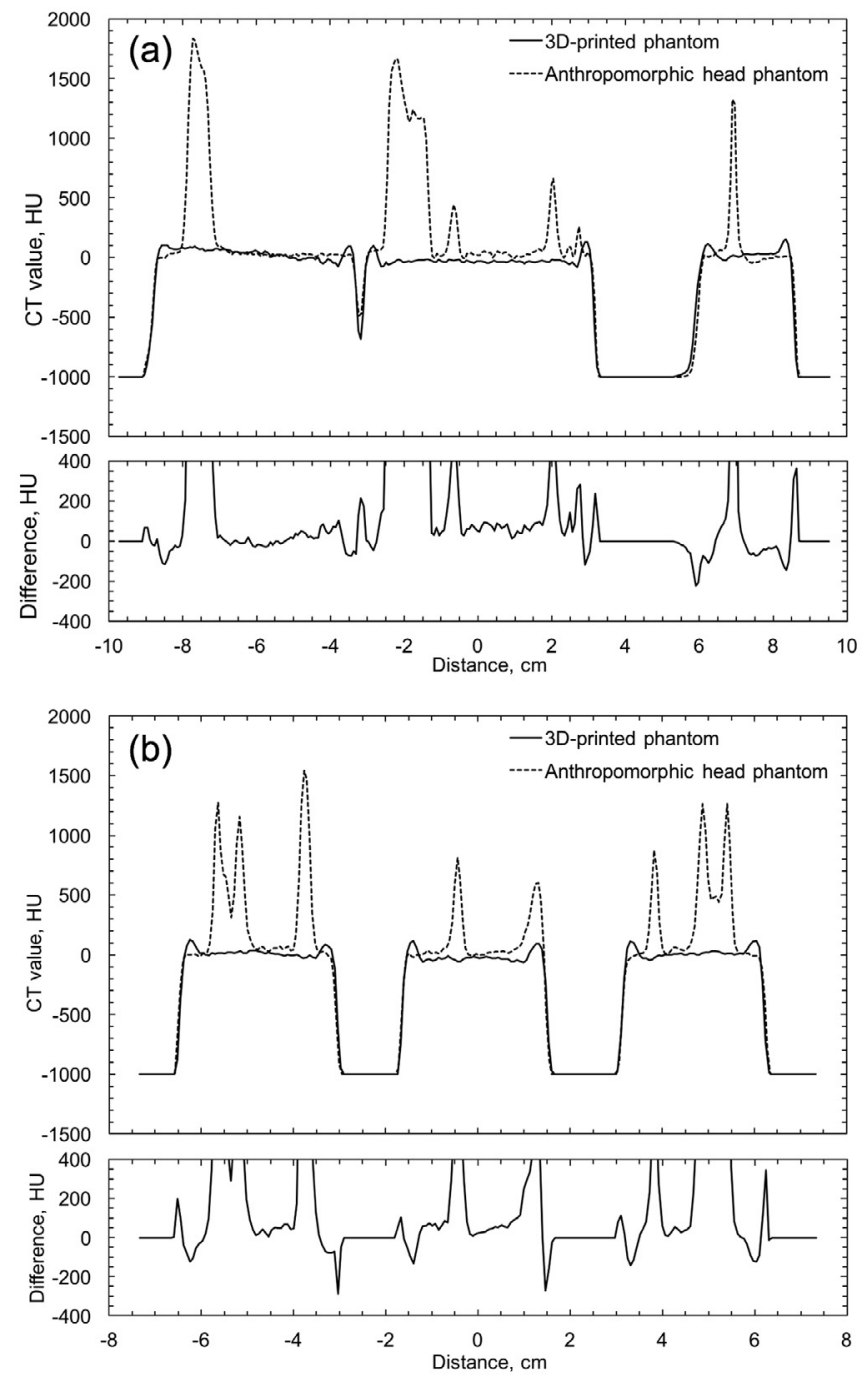

Fig. 6. CT value profiles and their differences along the white dotted arrows in Fig. $5 \mathrm{a}$ in the (a) sagittal and (b) coronal directions.

was $12.7 \pm 17.5 \mathrm{HU}$. The average CT value of the infill region in the printed phantom was $-5.8 \pm 18.8 \mathrm{HU}$ and that of the vertical shell region was $126.1 \pm 17.5 \mathrm{HU}$.

The measured dose differences between the anthropomorphic head phantom and the 3D-printed phantom are presented in Table 2. For single static field irradiation, the measured dose difference between the $3 \mathrm{D}$-printed and anthropomorphic phantoms was $0.6 \pm 1.9 \%$; for the two-arc VMAT plan, the measured dose difference was $1.5 \pm 1.2 \%$. The measured doses on the 3D-printed phantom were slightly increased compared to those of the anthropomorphic phantom.
The percentage dose differences between the measured and TPScalculated doses (measurement - calculation) relative to the TPS-calculated dose are indicated in Table 3. For single static field irradiation, the average percentage dose differences of the anthropomorphic head phantom, 3D-printed phantom, and 3D-printed phantom with converted CT values were $0.9 \pm 2.5 \%, 2.3 \pm 1.1 \%$, and $6.3 \pm 4.5 \%$, respectively. These average percentage dose differences did not include the results of the dosimeter location number seven, which was outside the radiation field. For the two-arc VMAT plan, the average percentage dose difference of the anthropomorphic head phantom, 3D-printed phantom, and 3D-printed phantom with converted CT values were $-1.2 \pm 1.6 \%,-2.1 \pm 1.9 \%$, and $1.3 \pm 2.7 \%$, respectively. All percentage dose differences between the anthropomorphic head phantom and 3D-printed phantom were within $\pm 5 \%$. However, the results of the 3D-printed phantom with converted CT values were poorer than those without converted CT values.

\section{Discussion}

Objects printed using FDM tend to warp during cooling, a characteristic that may depend on the extruder temperature, bed temperature, object size, area, and printing material. Several previous works had employed ABS filament because its physical properties closely resemble those of water $[17,25,26]$. However, ABS filament can undergo considerable deformation during cooling $[16,18,19]$; thus, we employed PLA filament. The results of this study demonstrate slight geometric errors between the CT scanned image of the anthropomorphic head phantom and the 3D-printed phantom, mostly in the bottom region. This geometric error also contains the uncertainty of the partial volume effect in the CT images. Except for the bottom region, the geometric error was less than $1 \mathrm{~mm}$. In addition, the Dice similarity coefficient for the external shape was equivalent to that in Ehler's work [17]. Thus, with respect to the geometrical reproducibility of the 3Dprinted phantom, it would be possible to use a patient-specific 3Dprinted phantom created using a PLA filament for radiotherapy dosimetry. Moreover, fabrication quality can be improved by optimizing the printing parameters and employing high-performance devices and software.

The average $\mathrm{HU}$ of the 3D-printed phantom infill region in this study is $-6 \pm 18 \mathrm{HU}$, lower than those in the previous works. Burleson et al. found the average HU of a 3D-printed PLA bolus to be approximately $160 \mathrm{HU}$ [26], whereas, Zue et al. declared it to be approximately 106 $\mathrm{HU}$ [27]. In contrast, the HU values of the vertical shell region in this work are approximately $125 \mathrm{HU}$, which is comparable to those in previous work $[26,27]$. This tendency resembles the control results of the fill density parameter reported by Madamesila et al. [28]. The most obvious cause of this change in $\mathrm{HU}$ seems to be the variation in the actual fill density, since the material composition of the infill and shell region are identical. Therefore, different 3D printers and software packages may produce different actual material densities for the same fill density parameter, and it is important to evaluate the actual fill

Table 2

Measured dose differences between the anthropomorphic head phantom and the 3D-printed phantom.

\begin{tabular}{|c|c|c|c|c|c|c|}
\hline \multirow[t]{2}{*}{ Location } & \multicolumn{3}{|l|}{ Single static field } & \multicolumn{3}{|l|}{ Two-arc VMAT plan } \\
\hline & $\begin{array}{l}\text { Anthropomorphic } \\
\text { (Gy } \pm 1 \mathrm{SD})\end{array}$ & $\begin{array}{l}\text { 3D-printed } \\
(\mathrm{Gy} \pm 1 \mathrm{SD})\end{array}$ & $\begin{array}{l}\text { Dose difference } \\
(\%)\end{array}$ & $\begin{array}{l}\text { Anthropomorphic } \\
\text { (Gy } \pm 1 S D)\end{array}$ & $\begin{array}{l}\text { 3D-printed } \\
(\mathrm{Gy} \pm 1 \mathrm{SD})\end{array}$ & $\begin{array}{l}\text { Dose difference } \\
(\%)\end{array}$ \\
\hline 1 & $1.01 \pm 0.013$ & $1.03 \pm 0.019$ & 1.6 & $1.91 \pm 0.019$ & $1.93 \pm 0.026$ & 0.8 \\
\hline 2 & $0.81 \pm 0.006$ & $0.83 \pm 0.007$ & 2.1 & $1.92 \pm 0.008$ & $1.93 \pm 0.033$ & 0.4 \\
\hline 3 & $0.67 \pm 0.001$ & $0.66 \pm 0.010$ & -1.2 & $1.02 \pm 0.010$ & $1.02 \pm 0.014$ & 0.3 \\
\hline 4 & $1.03 \pm 0.004$ & $1.01 \pm 0.006$ & -2.0 & $1.96 \pm 0.018$ & $2.00 \pm 0.016$ & 1.9 \\
\hline 5 & $0.79 \pm 0.011$ & $0.80 \pm 0.003$ & 1.0 & $1.97 \pm 0.005$ & $1.99 \pm 0.018$ & 1.3 \\
\hline 6 & $0.60 \pm 0.005$ & $0.62 \pm 0.005$ & 3.4 & $0.78 \pm 0.003$ & $0.79 \pm 0.017$ & 1.6 \\
\hline 7 & $0.03 \pm 0.000$ & $0.03 \pm 0.001$ & -0.3 & $1.04 \pm 0.031$ & $1.08 \pm 0.015$ & 3.9 \\
\hline
\end{tabular}


Table 3

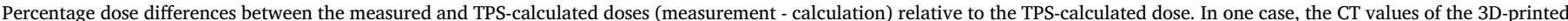
phantom were converted to the corresponding value of the PLA density of $1.25 \mathrm{~g} / \mathrm{cm}^{3}$.

\begin{tabular}{|c|c|c|c|c|c|c|}
\hline \multirow[t]{2}{*}{ Location } & \multicolumn{3}{|l|}{ Single static field } & \multicolumn{3}{|l|}{ Two-arc VMAT plan } \\
\hline & Anthropomorphic & 3D-printed & 3D-printed $\left(1.25 \mathrm{~g} / \mathrm{cm}^{3}\right)$ & Anthropomorphic & 3D-printed & 3D-printed $\left(1.25 \mathrm{~g} / \mathrm{cm}^{3}\right)$ \\
\hline 1 & -3.0 & 1.0 & 0.8 & -2.5 & -1.5 & 1.1 \\
\hline 2 & 0.6 & 2.3 & 5.5 & -0.1 & -1.7 & 2.9 \\
\hline 3 & 4.8 & 3.7 & 10.1 & -2.3 & -3.8 & -2.0 \\
\hline 4 & 0.5 & 0.9 & 2.0 & -1.0 & -2.0 & 2.4 \\
\hline 5 & 1.2 & 2.5 & 7.2 & 0.2 & -1.6 & 4.0 \\
\hline 6 & 1.3 & 3.1 & 12.3 & 0.9 & 1.0 & 3.5 \\
\hline 7 & 28.4 & 28.0 & 17.3 & -3.3 & -4.9 & -3.0 \\
\hline
\end{tabular}

density of each 3D printer and software package before using a 3Dprinted phantom for dosimetry.

RPL glass dosimeters were employed in this study. To minimize dose uncertainty caused by angular dependency, energy dependency, and fading, we carefully handled the dosimeters throughout, with particular adherence to previous studies [20,23]. In Japan, postal dose audits have been performed on radiation therapy units using RPL glass dosimeters since 2007 [29] and previous studies have employed RPL glass dosimeters for radiotherapy dose verification. Hus et al. compared measurements from an RPL glass dosimeter with those from a thermoluminescent dosimeter and radiographic film, and found differences less than $5 \%$, which is clinically acceptable accuracy [30]. Nakaguchi et al. also reported that the accuracy of the RPL glass dosimeter measurement was almost $5 \%$ and that angular dependency and geometric uncertainties should be considered [23]. They also raised the issue of geometric uncertainties in which the insertion slots in the phantom were larger than the RPL glass dosimeter. This is an advantage of 3Dprinted phantoms, in that they may reduce the geometric uncertainty caused by a diameter mismatch between the slots and the dosimeter.

In our environment, the maximum dose differences between the anthropomorphic head phantom and the 3D-printed phantom were $3.9 \%$, and most of the measurement points agreed within $2 \%$. As per our expectations, notable dose differences should have been obtained because this study employed PLA filament. However, the dose difference between the anthropomorphic head phantom and the 3D-printed phantom was almost within the measurement uncertainty for the RPL glass dosimeter $[23,30]$. A factor contributing to this result may be the low CT value in the infill region of the 3D-printed phantom, as discussed in the previous section. Differences in the equipment and software used may affect this result; thus, it is necessary to verify it in each environment. Moreover, the dosimetric impact of bone structures, described in previous studies [31,32], was not noticeable from the dose differences between the anthropomorphic head phantom and the 3Dprinted phantom. Ehler et al. also described difference in doses at a bone-tissue interface [15]. As for the necessity of considering bone structure, we agree with previous studies, and future investigation is needed. One potential reason for the low sensitivity of the dosimetric impact of the bone structure in this study is the energy dependency of the RPL glass dosimeter. The RPL glass dosimeter has a higher response to low-energy photons $[33,34]$. It is possible that because of extra lowenergy scattered photons and electrons inside the bone material [32], the doses measured by the RPL glass dosimeter were inaccurately accounted for by energy dependency.

Dosimetric comparison between the calculated TPS and the measured dose was carried out in this study, and the percentage dose differences between the anthropomorphic head phantom and 3D-printed phantom for single static field and VMAT plans were within $\pm 5 \%$, which is within the measurement uncertainty for the RPL glass dosimeter $[21,28]$. Note that measured doses were slightly lower in the VMAT cases than in the static field cases. We consider that this tendency may be caused by field-size dependence [33,34]. The average field size decreases by VMAT, low-energy scattered radiations in the phantom decreases, and the outputs of the RPL glass dosimeters decrease. Therefore, the field-size correction factor described by Mizuno et al. [33] may be useful for obtaining more accurate IMRT and VMAT treatment plan verification results. In many cases, when comparing the TPS-calculated and measured doses, the phantom density should be overridden in the TPS with a suitable value [26]. In this study, the CT values of the 3D-printed phantom was converted to the corresponding PLA density value of $1.25 \mathrm{~g} / \mathrm{cm}^{3}$. However, the results of the 3D-printed phantom with the converted CT values deteriorated compared to those without the converted CT values. In our experimental environment, converting the CT values with the PLA density can be regarded as overcorrection. This tendency does not contradict the result of low CT value in the infill region of the 3D-printed phantom, as mentioned in the above section.

The initial cost of the 3D printer used in this work was $\$ 2500$ and that of the filament was $\$ 40$ per $750 \mathrm{~g}$. This system, for producing patient-specific 3D-printed phantoms, was confirmed to have permissible geometric accuracy and is cost effective. However, the total printing time to fabricate the part was approximately $20 \mathrm{~h}$, which needs improvement. If the extruder head speed is identical, fabrication time depends on the distance that the extruder head moves. One of the methods for reducing fabrication time involves the usage of larger nozzle diameters (e.g. $0.6 \mathrm{~mm}$ ). Accordingly, it would be possible to use greater layer-height and extrusion-width settings to reduce the distance that the extruder head moves. However, this may affect the geometric fidelity and dosimetric properties of the phantom and requires additional evaluation.

\section{Conclusion}

We have constructed a system for producing patient-specific phantoms, using a low-cost personal 3D printer with a PLA filament. The modeling accuracy of the 3D-printed phantom was acceptable. The dose differences between the anthropomorphic head phantom and the 3Dprinted phantom were approximately within $2 \%$, in our experimental environment. These results demonstrate the feasibility of the 3Dprinted phantom for artificial in vivo dosimetry in radiotherapy QA.

\section{Acknowledgment}

The authors would like to thank Mr. Mukoyama Takashi and Mr. Kazuma Sugita (Nagoya University Hospital, Japan) for technical support during the phantom irradiation processes. This work was presented in part at the 22nd International Conference on Medical Physics (ICMP), Bangkok, Thailand, December 9-12, 2016. This research was supported by JSPS KAKENHI Grant Numbers JP15K21060 and JP17K15800. The funding agency has no role in the study design, data collection and analysis, decision to publish, and preparation of the manuscript. 


\section{References}

[1] Beadle BM, Liao KP, Elting LS, Buchholz TA, Ang KK, Garden AS, et al. Improved survival using intensity-modulated radiation therapy in head and neck cancers: a SEER-Medicare analysis. Cancer 2014;120:702-10.

[2] Mizowaki T, Norihisa Y, Takayama K, Ikeda I, Inokuchi H, Nakamura K, et al. Tenyear outcomes of intensity-modulated radiation therapy combined with neoadjuvant hormonal therapy for intermediate- and high-risk patients with T1c-T2NOM0 prostate cancer. Int J Clin Oncol 2016;21:783-90.

[3] Kamomae T, Itoh Y, Okudaira K, Nakaya T, Tomida M, Miyake Y, et al. Dosimetric impact of dental metallic crown on intensity-modulated radiotherapy and volumetric-modulated arc therapy for head and neck cancer. J Appl Clin Med Phys 2016;17:234-45.

[4] Kubo K, Monzen H, Ishii K, Tamura M, Kawamorita R, Sumida I, et al. Dosimetric comparison of RapidPlan and manually optimized plans in volumetric modulated arc therapy for prostate cancer. Physica medica: PM: an international journal devoted to the applications of physics to medicine and biology: official journal of the Italian Association of Biomedical Physics. 2017.

[5] Clinical Dosimetry Measurements in Radiotherapy. In: Rogers DWO, Cygler JE, editors. College Park, MD: American Association of Physicists in Medicine; 2009.

[6] Prescribing, Recording, and Reporting Photon-Beam Intensity-Modulated Radiation Therapy (IMRT): Contents. Journal of the ICRU. 2010;10.

[7] Low DA, Moran JM, Dempsey JF, Dong L, Oldham M. Dosimetry tools and techniques for IMRT. Med Phys 2011;38:1313-38.

[8] ESTRO Booklet 9: Guidelines for the Verification of IMRT. Brussels: ESTRO; 2008.

[9] Ezzell GA, Burmeister JW, Dogan N, LoSasso TJ, Mechalakos JG, Mihailidis D, et al. IMRT commissioning: multiple institution planning and dosimetry comparisons, a report from AAPM Task Group 119. Med Phys 2009;36:5359-73.

[10] Zani M, Bucciolini M, Casati M, Talamonti C, Marinelli M, Prestopino G, et al. A synthetic diamond diode in volumetric modulated arc therapy dosimetry. Med Phys 2013;40:092103.

[11] Kamomae T, Oita M, Hayashi N, Sasaki M, Aoyama H, Oguchi H, et al. Characterization of stochastic noise and post-irradiation density growth for reflective-type radiochromic film in therapeutic photon beam dosimetry. Phys Medica 2016;32:1314-20.

[12] Nelms BE, Zhen H, Tome WA. Per-beam, planar IMRT QA passing rates do not predict clinically relevant patient dose errors. Med Phys 2011;38:1037-44.

[13] Zhen H, Nelms BE, Tome WA. Moving from gamma passing rates to patient DVHbased QA metrics in pretreatment dose QA. Med Phys 2011;38:5477-89.

[14] Fujimoto K, Shiinoki T, Yuasa Y, Hanazawa H, Shibuya K. Efficacy of patient-specific bolus created using three-dimensional printing technique in photon radioherapy. Phys Medica 2017:38:1-9.

[15] Ju SG, Kim MK, Hong CS, Kim JS, Han Y, Choi DH, et al. New technique for developing a proton range compensator with use of a 3-dimensional printer. Int $J$ Radiat Oncol 2014;88:453-8.

[16] Park K, Park S, Jeon MJ, Choi J, Kim JW, Cho YJ, et al. Clinical application of 3Dprinted-step-bolus in post-total-mastectomy electron conformal therapy. Oncotarget. 2016

[17] Ehler ED, Barney BM, Higgins PD, Dusenbery KE. Patient specific 3D printed phantom for IMRT quality assurance. Phys Med Biol 2014:59:5763-73.

[18] Harris BD, Nilsson S, Poole CM. A feasibility study for using ABS plastic and a low cost 3D printer for patient-specific brachytherapy mould design. Australas Phys Eng S 2015;38:399-412.

[19] Chilson L. The Difference Between ABS and PLA for 3D Printing. http://www protoparadigm.com/news-updates/the-difference-between-abs-and-pla-for-3dprinting/2013.

[20] Arakia F, Moribe N, Shimonobou T, Yamashita Y. Dosimetric properties of radiophotoluminescent glass rod detector in high-energy photon beams from a linea accelerator and cyber-knife. Med Phys 2004;31:1980-6.

[21] Rah JE, Hong JY, Kim GY, Kim YL, Shin DO, Suh TS. A comparison of the dosimetric characteristics of a glass rod dosimeter and a thermoluminescent dosimeter for mailed dosimeter. Radiat Meas 2009;44:18-22.

[22] Ranellucci A. Slic3r. http://slic3r.org/.

[23] Nakaguchi Y, Ono T, Maruyama M, Nagasue N, Shimohigashi Y, Kai Y. Validation of fluence-based 3D IMRT dose reconstruction on a heterogeneous anthropomorphic phantom using Monte Carlo simulation. J Appl Clin Med Phys/Am College Med Phys 2015;16:5199.

[24] Dice LR. Measures of the amount of ecologic association between species. Ecology $1945 ; 26: 297-302$.

[25] Kumar R, Sharma SD, Deshpande S, Ghadi Y, Shaiju VS, Amols HI, et al. Acrylonitrile Butadiene Styrene (ABS) plastic based low cost tissue equivalent phantom for verification dosimetry in IMRT. J Appl Clin Med Phys/Am College Med Phys 2009;11:3030.

[26] Burleson S, Baker J, Hsia AT, Xu ZG. Use of 3D printers to create a patient-specific 3D bolus for external beam therapy. J Appl Clin Med Phys 2015;16:166-78.

[27] Zou W, Fisher T, Zhang M, Kim L, Chen T, Narra V, et al. Potential of 3D printing technologies for fabrication of electron bolus and proton compensators. J Appl Clin Med Phys 2015;16:90-8.

[28] Madamesila J, McGeachy P, Barajas JEV, Khan R. Characterizing 3D printing in the fabrication of variable density phantoms for quality assurance of radiotherapy. Phys Medica 2016:32:242-7.

[29] Mizuno H, Kanai T, Kusano Y, Ko S, Ono M, Fukumura A, et al. Feasibility study of glass dosimeter postal dosimetry audit of high-energy radiotherapy photon beams. Radiother Oncol 2008;86:258-63.

[30] Hsu SM, Hung CH, Liao YJ, Fu HM, Tsai JT, Huang YH, et al. Feasibility study on applying radiophotoluminescent glass dosimeters for cyberknife SRS dose verification. PLoS ONE 2017;12:e0169252.

[31] Ma CM, Jiang SB, Pawlicki T, Chen Y, Li JS, Deng J, et al. A quality assurance phantom for IMRT dose verification. Phys Med Biol 2003;48:561-72.

[32] van Elmpt WJC, Nijsten SMJJG, Dekker ALAJ, Mijnheer BJ, Lambin P. Treatment verification in the presence of inhomogeneities using EPID-based three-dimensional dose reconstruction. Med Phys 2007;34:2816-26.

[33] Mizuno H, Fukumura A, Fukahori M, Sakata S, Yamashita W, Takase N, et al Application of a radiophotoluminescent glass dosimeter to nonreference condition dosimetry in the postal dose audit system. Med Phys 2014;41:112104.

[34] Araki F, Ohno T. The response of a radiophotoluminescent glass dosimeter in megavoltage photon and electron beams. Med Phys 2014;41:122102. 